\title{
Improved thermal energy storage for heating and cooling of buildings
}

\author{
Rok Koželj, Žiga Ahčin, Eva Zavrl, and Uroš Stritih \\ University of Ljubljana, Faculty of Mechanical Engineering, Aškerčeva 6, 1000 Ljubljana, Slovenia
}

\begin{abstract}
One of the great challenges in the energy sector represents retrofit of residential buildings where $3 / 4$ of buildings in Europe are residential. To reduce energy consumption and increase the use of renewables in existing residential buildings a holistic approach of retrofit with interconnected technological system is needed. In the present paper energy toolkit based on the synergetic interaction between technologies integrated in the system for holistic retrofit of residential buildings which is under development within HEART project (HORIZON 2020) is presented. In this project step towards self-sufficient heating and cooling of building is made with an increase in on-site consumption of self-produced energy in PV from solar energy, where produced electrical energy is used also for heat pump operation. In this case thermal energy storage plays an important role for storing heat or cold for time when solar energy is not available. Improvement of sensible thermal energy storage with implemented cylindrical modules at the top of the heat storage tank and filled with phase change material is investigated experimentally. 43 litres of paraffin with phase change temperature between $27^{\circ} \mathrm{C}$ and $29^{\circ} \mathrm{C}$ was used in a system, what represented $15 \%$ of total volume of heat storage tank. The results from experiment shows that thermal energy storage unit with integrated modules filled with phase change material can supply desired level of water temperature for twice as long at smaller temperature level as sensible thermal energy storage what is the consequence of higher energy density that can be stored during phase change. The advantage of phase change materials is in thermal energy storage for applications that needs narrow temperature range of supplying and storing thermal energy what is the subject matter of consideration in the case of HEART project.
\end{abstract}

\section{Introduction}

Prudent and efficient utilization of renewable energy sources is needed in order to meet demands from European directive on the energy performance of buildings (EPBD) and to comply with Paris agreement for global clean energy transition [1]. To reduce final energy use in newly constructed buildings is one thing but it represents a great challenge in existing ones.

Ever since the researchers on renewable energy field have been encouraged with European directive, improvement on utilizing renewables has been reached. However the challenge in utilizing renewable energy sources is in its time unsteadiness when mismatch between production and demand occurs. With thermal energy storage integration into system with renewable energy source utilization, it is possible to levelize mentioned mismatch between production from renewables and demand for heating or cooling of buildings. In a present paper the focus is on investigating the possibility for enhancing energy density in thermal energy storage application for heating and cooling of residential buildings. The system in which thermal energy storage is considered, represents heating and cooling system in residential buildings that will be renovated. Heating and cooling system consists of
DC heat pump as main thermal energy source which obtain power supply from PV and electrical energy storage. Because both sources are highly dependent on weather conditions, this represents the reason that thermal energy storage is implemented into the considered system owing to the fact that it balances the mismatch between supply and demand. The whole considered system which is developed within EU project HEART is presented in the next section. However the emphasis in the present paper is made on the research of sensible thermal energy storage improvement with phase change materials (PCMs).

A number of research have already been carried out in this field and many prototypes have been developed. An extended research has been done within IEA Solar Heating and Cooling Programme under Task 32 Advanced storage concepts [2]. During this researches several prototypes of heat storage with a use of PCM has been tested in solar combisystems for single family houses in order to provide space heating and domestic hot water. It was found out that for small temperature differences of $20 \mathrm{~K}$ and with a fin and tube heat exchanger that is immersed in the PCM storage tank, can be sized for about $1 / 3$ of the volume compared to water only store unit by using sodium acetate trihydrate. However for the same

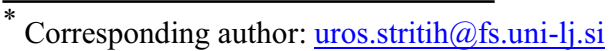


PCM material but macro-encapsulated and for the temperature lift of $50 \mathrm{~K}$ and $60 \mathrm{~K}$ in solar combisystems, the PCM storage unit has the same size as a water store.

Mehling et al. [3] studied the impact of the integration of PCM modules at the top of the water heat storage tank and found out that this improves storage density by 20 to 45 $\%$, allowing reheating of the transition layer after partial unloading and thus reducing heat losses.

In the research of Bourne et al. [4] the latent thermal energy storage system compared to a similar-performing chilled water tank was investigated for improved energy efficiency of a chiller by shifting cooling load to non-peak electric energy loads. It was found that encapsulated tetradecane phase change material in tubes submerged in water-glycol tank can reduce size of a chilled water tank by more than a factor 2 .

Experimental research of an air-source heat pump water heater using water-PCM for thermal storage has been done by Zou et al. [5], where the heat storage is increased by $14 \%$ compared to the standalone sensible heat storage just by adding PCMs around condenser coil.

According to the Abdelsalam et al. [6] the energy density of thermal storage unit containing water with submerged PCMs can increase energy density of thermal storage from two to five times.

In research of Fang et al. [7] a tube-in-tank latent thermal storage unit was investigated, where PCM is surrounded around several tubes in tank through which heat transfer fluid flows in charging or discharging period. The effective energy storage capacity of mentioned latent thermal storage system can reach 4.4 times of that of an ideal stratified water storage system.

Lu et al. [8] proposed the improvement of buffer storage with multi-melting point PCMs. Investigated latent thermal storage unit where compared with the conventional sensible storage unit, where the PCM accounting for $19 \%$ of the tank volume would extend time of heat release by $34 \%$.

In research of de Cunha et al. [9] comparison between performance of conventional gas boiler and air source heat pump with latent thermal storage unit has been performed for a domestic space and hot water heating system with thermal solar collectors in a semi-detached dwelling. The latent thermal storage system with paraffin provided $53 \%$ of extra thermal storage capacity compared to a sensible thermal storage unit. According to simulation, yearly $\mathrm{CO}_{2}$ reduction potential is $56 \%$ and yearly energy reduction potential is $76 \%$.

From these researches it can be shortly summarized that PCMs in thermal storage applications offer a meaningful advantage compared to water thermal storages from energy density point of view. That also leads to extended discharging periods of thermal energy release according to the same volume as in case of water only store. Due to the aforementioned advantages, the present paper focuses on improvement of sensible thermal storage unit with
PCMs for highest possible utilization of solar energy for heating and cooling of residential buildings.

\section{HEART toolkit and system description}

The considered research of thermal energy storage is placed in a system which is under development within HEART consortium. HEART stands for Holistic Energy and Architectural Retrofit Toolkit. The project follows the goals of the European Union to reduce the consumption of primary resources by $80 \%$ by the end of 2050 , which can be achieved with nearly zero-energy buildings. Therefore, the project foresees the creation of a software toolkit that will enable a comprehensive energy and architectural renovation of buildings. The software toolkit will be used in all phases of the project, from conceptual design to management and maintenance of the building.

HEART is a multifunctional retrofit toolkit that includes different components in building retrofit process such as: ICT (Information and Communication Technology), BEMS (Building Energy Management Systems), HVAC (Heating, Ventilation and Air Conditioning), BIPV (Building Integrate Photovoltaic) and Envelope Technologies which operate synergistically to transform an existing building into a smart building. The toolkit runs on cloud-based platform with computing power, concentrating managing and operational logic that supports decision-making in the planning phase of buildings' energy retrofit and optimizes energy performance in the operational one.

The concept of HEART toolkit with included subcomponents and their connectivity in the system is presented in the Fig. 1. Those subcomponents are under development within the consortium of HEART project and are listed below:

- cloud-based DSS (Decision Support System) and BEMS (Building Energy Management System),

- multifunctional prefabricated external thermal insulation in which wiring, pipes, vents and sensing options can be integrated,

- universal PV tiles for roofs, customized with respect to common roofing,

- Multi-Input/Multi-Output power controller for interconnection optimization among production, electrical storage and consumption devices,

- hydronic air to water DC modular heat pump,

- dvanced hot/cold storage units for thermal energy storage of heat pump that runs on PV,

- battery pack for PV electrical energy and power management (the only subcomponent not specifically developed in the project),

- DC smart fan-coils for heating/cooling of rooms (replacing existing radiators) [10].

For efficient operation of the whole system every single part of the system is focused on during this project. However in the present study only experimental investigation of the thermal energy storage is presented. 


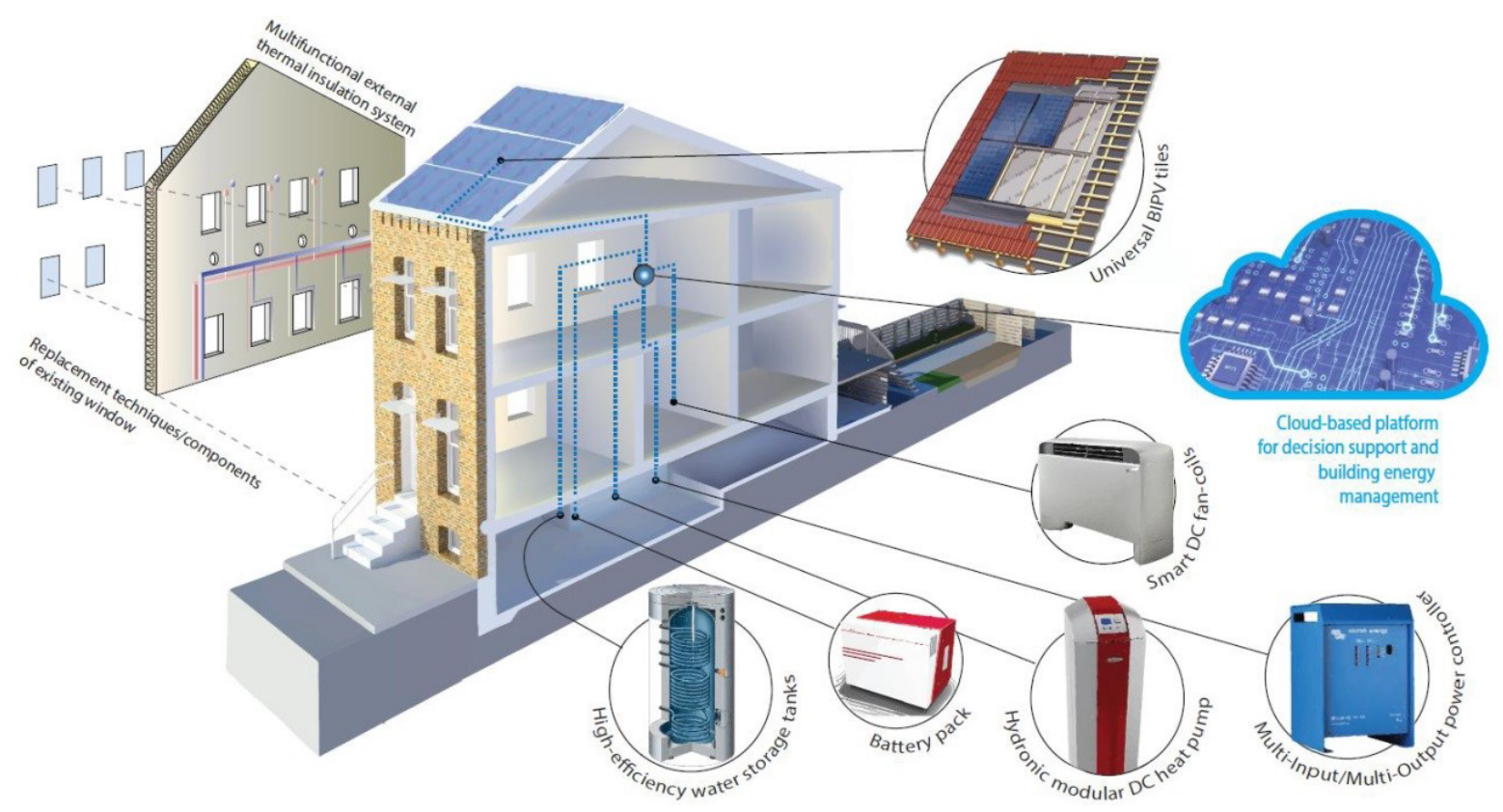

Fig. 1. Concept of HEART toolkit [10].

Nevertheless the interconnectivity and synergetic interaction between components are of significant importance that is why the summary of the system description has been introduced in this section.

\section{Thermal energy storage}

\subsection{Sensible thermal energy storage}

Sensible thermal energy storage is by far the most commonly used principle of thermal energy storage. Thermal energy is stored by changing the temperature of storage medium, for example water, sand, earth etc. The principle of thermal energy storage in this method is based on changing the temperature of the storage media without phase change. The amount of stored thermal energy is proportional to the temperature rise $(T)$, the specific heat capacity $\left(c_{\mathrm{p}}\right)$ and the mass of the medium $(m)$, as shown in Equation (1). The rise in temperature is a consequence of increased kinetic energy of the molecules. This change in internal energy of storage medium can be sensed by temperature sensors and consequently is called sensible heat [11].

$$
\Delta Q_{\mathrm{S}}=m \cdot c_{\mathrm{p}} \cdot \Delta T
$$

\subsection{Latent thermal energy storage}

In case of latent thermal energy storage, thermal energy is stored through phase change of storage medium. During phase change of medium thermal energy can be released at nearly constant temperature. These offers thermal storage densities 5 to 15 times greater compared to sensible thermal storages. Materials used in latent thermal storages are known as phase change materials (PCMs). The most common change of phase used in the system is solid - liquid, but there are also systems that uses solid gas, liquid - gas and solid - solid change of phase. The amount of stored thermal energy is proportional to the mass of the medium $(m)$ and the change of specific enthalpy $(\Delta h)$ between phases as shown in Equation (2) [11].

$$
\Delta Q_{\mathrm{L}}=m \cdot \Delta h
$$

\subsection{Working principle}

The Fig. 2 shows a comparison between sensible and latent principle of storing thermal energy. Below the temperature of phase change both materials heat up evenly when heat is supplied, assuming they have the same specific heat capacity. This increases kinetic energy of molecules or the oscillation of molecules. When the temperature of the phase change is reached, the temperature of the medium for latent thermal energy storage does not change when further heat is supplied. The heat input is reflected as an increased potential energy of the molecules, while the kinetic energy remains the same. After the phase change is completed, the kinetic energy of the molecules increases again with further heat supply [11].

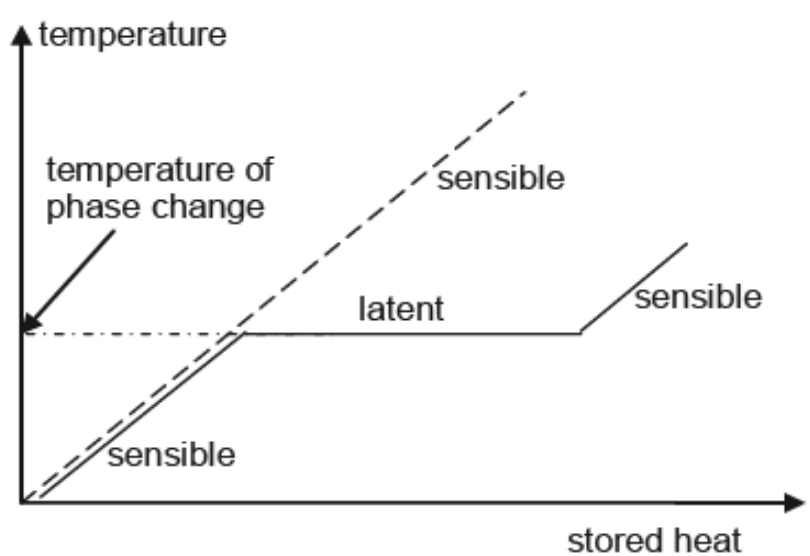

Fig. 2. Working principle of sensible \& latent heat storage [11]. 


\section{Experimental investigation}

The purpose of this experiment was to investigate improvement on increased energy density for thermal energy storage by integrating PCM modules in water storage tank. The influence of integrated modules on time of providing heat demand and the maximum heat output, has also been studied.

\subsection{Experimental system}

The experimental system was designed according to the standard VDI 2146 - PCM energy storage systems in building services [12], which includes basic definitions and calculation procedures for evaluating thermal energy storage systems with PCMs. It defines measurements of heating and cooling of storage via internal heat exchanger or direct double port connections and measurements of thermal energy storage in standby.

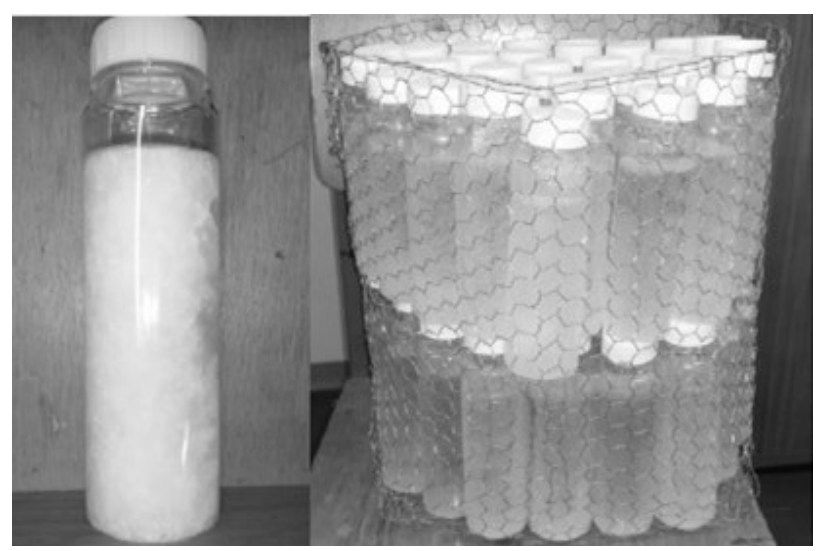

Fig. 3. Cylindrical modules filled with PCM.

The storage tank of volume $0.28 \mathrm{~m}^{3}$ was used. A large part of time has been dedicated to selection of the right shape of the modules. Important parameters in shape selection are available heat transfer area, which is crucial for the performance of the storage system, their sealing and mechanical resistance to prevent leakage and also price of the modules. There are different forms in which PCMs can be inserted into the storage tank, e.g. packs, panels, balls (spherical modules), cylindrical shape etc. According to the stated parameters, the cylindrical modules were chosen, as shown in Fig. 3. Each of them has a volume of $320 \mathrm{ml}$ and was filled with $250 \mathrm{ml}$ of PCM. This is due to thermal expansion of PCM when melted. PCM RT $28 \mathrm{HC}$ [13] was used which is paraffin with improved thermal capacitance of $250 \mathrm{~kJ} / \mathrm{kg}+/-7,5 \%$ and phase change temperature between $27^{\circ} \mathrm{C}$ and $29^{\circ} \mathrm{C}$ with main peak at $28^{\circ} \mathrm{C}$. Then a metal mesh was installed into the storage tank, in which the cylindrical modules with PCM were stacked. A total of 172 modules were integrated with total PCM volume of 431 , which represents $15 \%$ of total volume of the thermal energy storage tank.

For preparation of hot water, the electrical flow heat exchanger was used, which consists of two $2 \mathrm{~kW}$ electrical heaters. The mass flow rate was measured using flying start-stop method. Type $\mathrm{T}$ thermocouples were used for measuring temperature. The circulation pump has also been integrated in the experimental system to establish a uniform temperature inside the storage tank. Fig. 4 shows the experimental setup, where hydraulic connections and locations of temperature sensors, shut-off valves and circulation pump can be seen.

\subsection{Measurements}

Two sets of measurements were performed, namely measurements of the sensible and measurements of the latent thermal energy storage unit. The work focused on cooling the heat storage, as this is the most important part of operation of the heat storage.

The sensible thermal energy storage unit was heated up to an average temperature of 1 to $2 \mathrm{~K}$ higher than desired temperature, which was $40{ }^{\circ} \mathrm{C}$. Then the circulation pump was turn on until a uniform temperature inside the thermal energy storage unit was reached and desired temperature was established. In the next phase, cold water was supplied via direct double port connection in the bottom of the thermal energy storage unit, and consequently hot

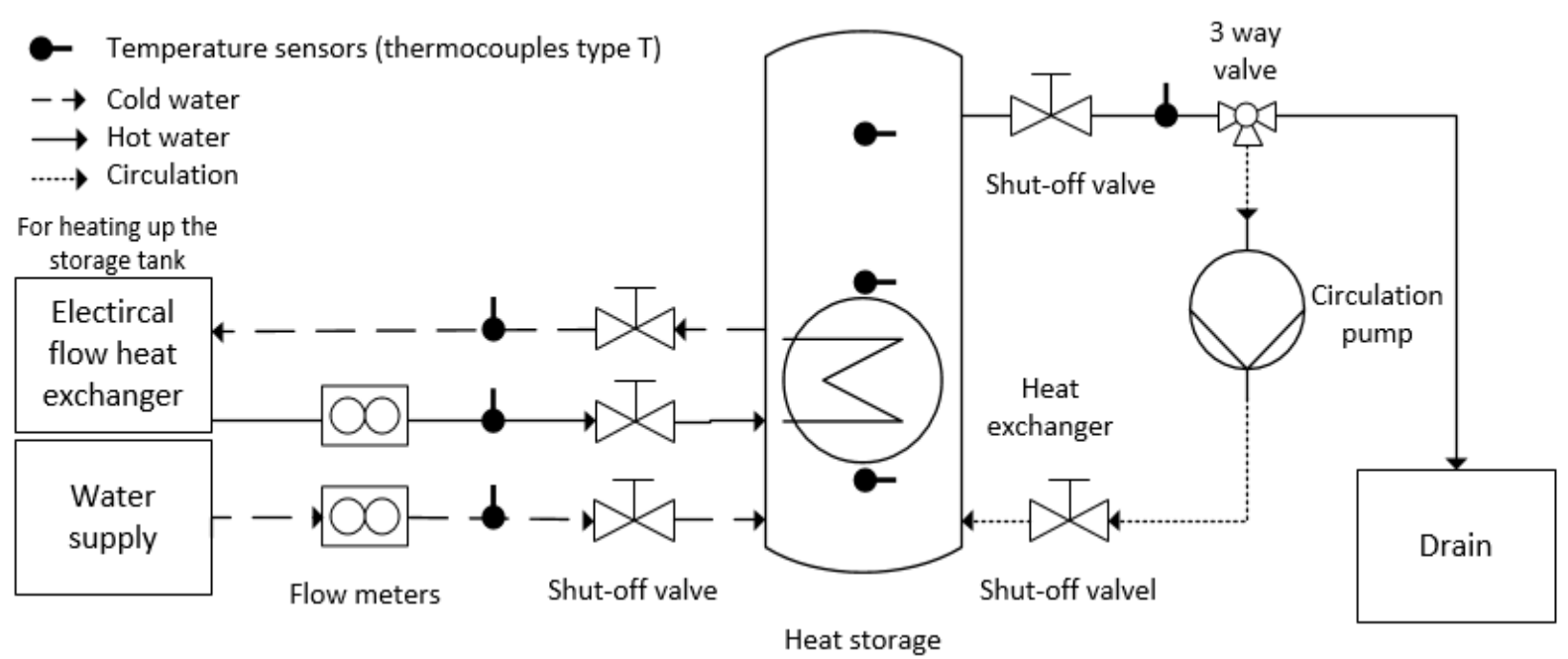

Fig 4. Scheme of experimental setup. 
water was exiting on the top of the thermal energy storage unit via direct double port connection.

In case of latent heat storage, a different approach was needed, since on the basis of a visual assessment it was not possible to assess whether the entire PCM was melted or not. The amount of energy required to complete the phase change was calculated. Accordingly, the initial water temperature in the thermal energy storage unit was determined, so that the entire PCM would melt and the thermal energy storage would have stayed heated to an average temperature of 1 to $2 \mathrm{~K}$ higher than desired temperature, which was $32{ }^{\circ} \mathrm{C}$. Then the procedure was repeated as in the case of sensible heat storage to achieve uniform temperature inside the thermal storage unit.

The temperature of supplied water was set to $5 \mathrm{~K}$ below phase change temperature. In this case this means $23{ }^{\circ} \mathrm{C}$, with maximum deviation of $0.33 \mathrm{~K}$. The water mass flow rate was set to $2 \mathrm{~kg} / \mathrm{min}$ with maximum deviation of 0.02 $\mathrm{kg} / \mathrm{min}$. The experiment was carried out under these conditions to the point where the temperature difference between the inlet and outlet water dropped below $0.5 \mathrm{~K}$.

\section{Results and discussion}

Fig. 5 and Fig. 6 shows the temperature distribution across the thermal energy storage unit. The proposed heating system requires a minimum inlet water temperature in the heating mode of $25^{\circ} \mathrm{C}$. This was also the point to which the time of covering the heat demand was measured. As it can be seen, the latent thermal energy storage unit may, despite the lower initial temperature, provide a minimum temperature of $25^{\circ} \mathrm{C}$ for longer period of time. Latent thermal energy storage unit can provide minimum outlet temperature of $25^{\circ} \mathrm{C}$ for $300 \mathrm{~min}$ while sensible one for $150 \mathrm{~min}$.

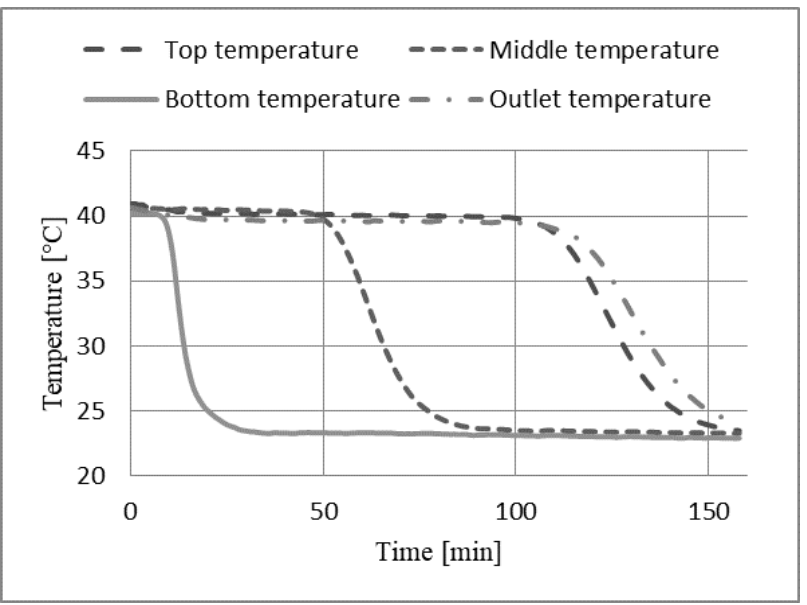

Fig. 5. Temperature distribution in case of sensible thermal storage unit.

Further on, temperature of the thermal energy storage according to stored thermal energy was calculated from Equations (3), (4) and (5), while the volume of the storage tank was assumed to be $1 \mathrm{~m}^{3}$. Equation (3) was used to calculate stored thermal energy in the sensible thermal energy storage unit. Equation (4) was used to calculate

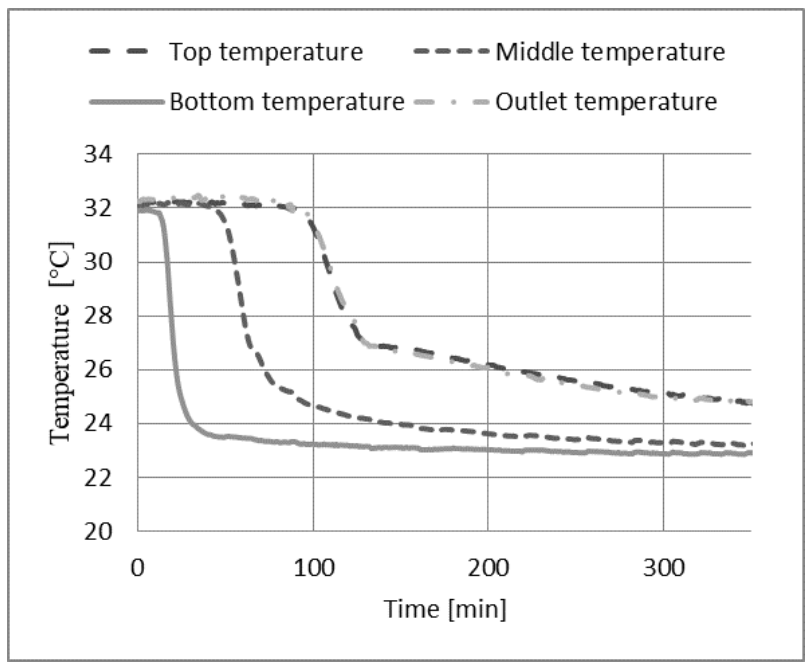

Fig. 6. Temperature distribution in case of latent thermal storage unit.

stored thermal energy in the latent thermal energy storage unit below the phase change temperature. Equation (5) was used for calculation of stored thermal energy in the latent thermal energy storage unit during phase change and above the phase change temperature. At the time when phase change took place constant temperature was assumed.

$$
\begin{gathered}
Q_{\mathrm{S}}=\rho_{\mathrm{w}} \cdot V_{\mathrm{w}} \cdot c_{\mathrm{p}, \mathrm{w}} \cdot\left(T_{\mathrm{s}}-T_{\mathrm{f}}\right) \\
Q_{\mathrm{L}}=\rho_{\mathrm{w}} \cdot V_{\mathrm{w}} \cdot c_{\mathrm{p}, \mathrm{w}} \cdot\left(T_{\mathrm{s}}-T_{\mathrm{f}}\right)+ \\
+\rho_{\mathrm{PCM}} \cdot V_{\mathrm{PCM}} \cdot c_{\mathrm{p}, \mathrm{PCM}} \cdot\left(T_{\mathrm{s}}-T_{\mathrm{f}}\right)
\end{gathered}
$$

$$
\begin{gathered}
Q_{\mathrm{L}}=\rho_{\mathrm{w}} \cdot V_{\mathrm{w}} \cdot c_{\mathrm{p}, \mathrm{w}} \cdot\left(T_{\mathrm{s}}-T_{\mathrm{f}}\right)+\rho_{\mathrm{PCM}} \cdot V_{\mathrm{PCM}} \cdot c_{\mathrm{p}, \mathrm{PCM}} \cdot \\
\cdot\left(T_{\mathrm{s}}-T_{\mathrm{f}}\right)+\rho_{\mathrm{PCM}} \cdot V_{\mathrm{PCM}} \cdot \Delta h
\end{gathered}
$$

Equation (3), (4) and (5) consists of following parameters: heat $(Q)$, density $(\rho)$, volume $(V)$, specific heat $(c)$, temperature $(T)$ and specific enthalpy $(h)$. The subscript $w$ is for water, $P C M$ is for phase change material, $s$ is for start and $f$ is for finish state.

Fig. 7 shows dependence of the temperature of the thermal energy storage fluid on the stored thermal energy in volume of $1 \mathrm{~m}^{3}$ for sensible and latent thermal energy storage unit at three different percentages of PCM in the unit. From results we see that the increase in the percentage of the PCM greatly lowers the temperature in the thermal energy storage unit. However, because of the lower specific heat of the PCM compared to water, this advantage is reduced by increasing the temperature in the thermal energy storage unit. Fig. 8 and Fig. 9 shows dependence of volume of the thermal energy storage unit on stored thermal energy at temperature difference of $5 \mathrm{~K}$ and $25 \mathrm{~K}$, respectively. As expected, the volume of the thermal energy storage unit is reduced by increasing the percentage of the PCM. This difference is reduced by increasing the temperature differences between the initial and final temperature when storing thermal energy. This 


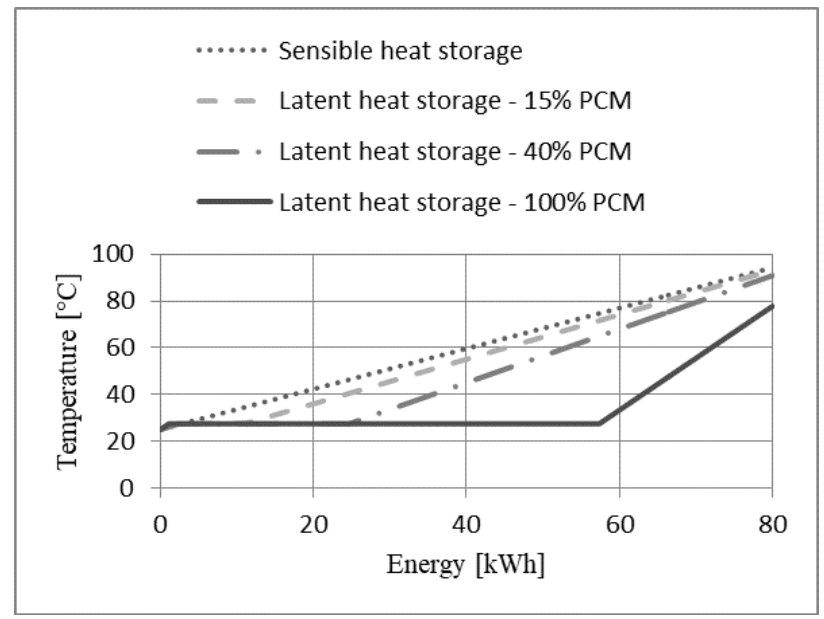

Fig. 7. Temperature of thermal storage unit according to stored thermal energy.

also indicates that thermal energy storage with PCMs have great advantage at smaller temperature difference of operating system compared to sensible one.

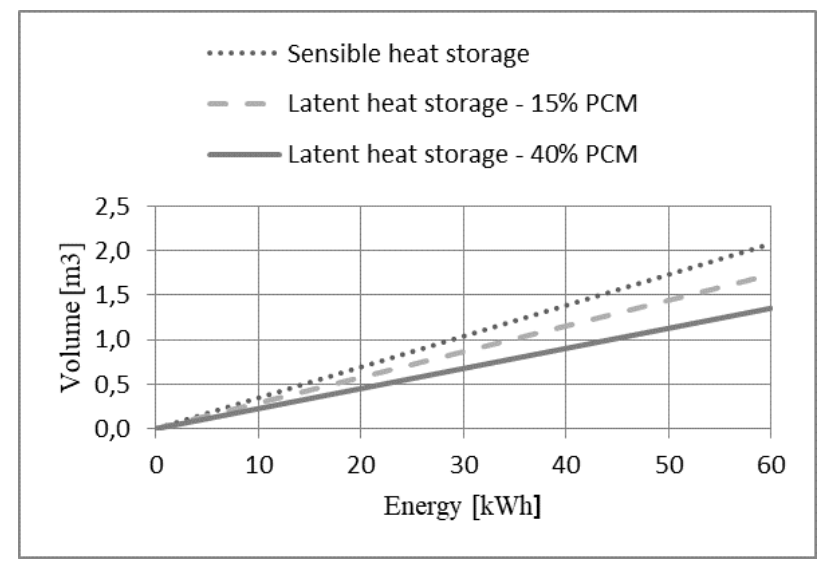

Fig. 8. Volume of thermal storage unit according to stored thermal energy at temperature difference $5 \mathrm{~K}$.

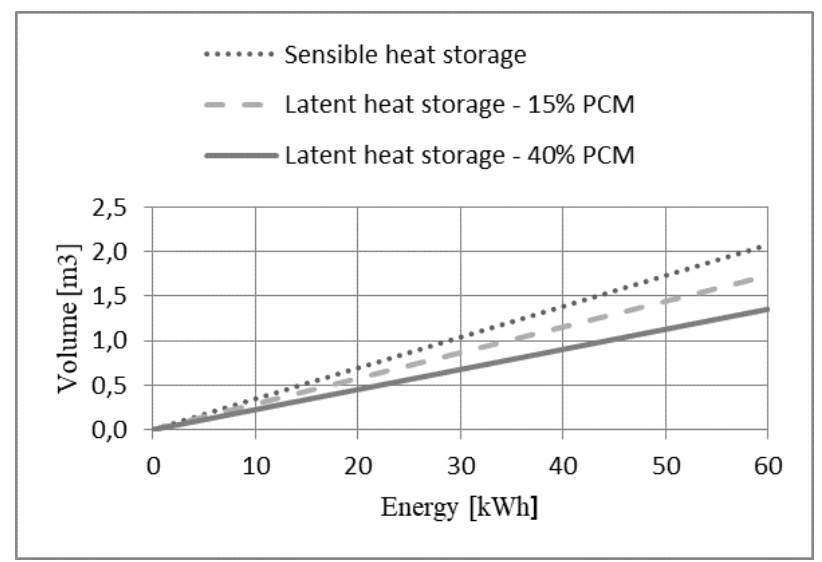

Fig. 9. Volume of thermal storage unit according to stored thermal energy at temperature difference $25 \mathrm{~K}$.

According to the results shown on Fig. 5-9 one can decide between two options. Therefore by integrating modules with PCM, the temperature of thermal energy storage unit can be greatly reduced and energy density of stored thermal energy can be increased at the same volume of the thermal energy storage unit. The other option is that total volume of the thermal energy storage unit can be decreased and the same temperature level is maintained in comparison to the sensible thermal energy storage unit.

\section{Conclusion}

The paper focuses on improvement of sensible thermal energy storage with PCMs where its main advantages have been reviewed and researched. The comparison between sensible and latent thermal energy storage unit have been carried out experimentally. From obtained results it have been found out that thermal energy storage unit with only $15 \%$ of encapsulated PCM submerged in water (Fig. 6) extends heat supply for two times longer than the sensible thermal energy storage unit (Fig. 5).

From thermodynamic point of view it is obvious that latent thermal energy storage have advantage over sensible thermal storage. However as seen from analytical and experimental results in present paper the comparison between them depends mainly on the temperature difference between the initial and final temperature when storing thermal energy. That is why the main advantage of the latent thermal energy storage system is in small temperature difference of storing thermal energy. This advantage is reduce when temperature difference arises and despite that latent thermal energy storage still has advantage over sensible one. However its installation in the system make it less feasible from economical point of view because the price of water in comparison to PCMs is incomparable.

One of the advantages where can latent thermal energy storage unit come into consideration are the systems with really high temperature levels. This is the case of biomass boilers where thermal energy storage units are heated up to $85{ }^{\circ} \mathrm{C}$. In this case latent thermal energy storage unit can store higher amount of thermal energy per unit volume because the sensible thermal storage unit is not heated on a much higher temperature level.

Another advantages of installing latent thermal energy storage units are systems where temperature source is limited upwards and storage of thermal energy at small temperature level with small temperature difference is needed (i.e. cooling system and low temperature heating system). With this we also obtain higher thermal energy storage density which is especially advantageous in case where we are dealing with a lack of space. The advantages of using latent thermal energy storage unit are reflected primarily in combinations with heat pumps, where more constant temperature conditions allow the operation of the heat pump in more stable conditions, which is reflected in achieving better efficiency of operation.

Synopsis of the present research can be concluded that PCMs have been recognized as potential advantage in considered system of HEART project where small temperature differences and low temperature level is the 
subject of matter. In conclusion a remark should be noted that the price plays a significant role of using PCMs as thermal energy storage. This should be taken into account in the further research.

This work was supported by EU Research and Innovation programme Horizon 2020 trough project number 768921 HEART.

\section{References}

1. Communication from the Commission to the European parliament and the council, The Road from Paris, European Commission, (2016)

2. J. M. Schultz, C. Sole, L. Cabeza, J. Bony, A. Heinz, W. Streicher, Laboratory Prototypes of PCM Storage Units, Task 32, (2007)

3. H. Mehling, L. F. Cabeza, S. Hippeli, S. Hiebler, PCM module to improve hot water heat stores with stratification, Elsevier Science Ltd., (2003)

4. S. Bourne, A. Novoselac, PCM-based High-density Thermal Storage Systems for Residential and Small Commercial Retrofit Applications, Procedia Eng.

121, 536-543 (2015)

5. D. Zou, X. Ma, X. Liu, P. Zheng, B. Cai, J. Huang, M. Liu, Experimental research of an air-source heat pump water heater using water-PCM for heat storage, App. Energy 206, 784-792 (2017)

6. M. Y. Abdelsalam, P. Sarafraz, J.S. Cotton, M. F. Lightstone, Heat transfer characteristics of a hybrid thermal energy storage tank with Phase Change Materials (PCMs) during indirect charging using isothermal coil heat exchanger, Solar Energy 157, 462-476 (2017)

7. Y. Fang, J. Niu, S. Deng, Numerical analysis for maximizing effective energy storage capacity of thermal energy storage systems by enhancing heat transfer in PCM, Energy and Buildings 160, 10-18 (2018)

8. S. Lu, T. Zhang, Y. Chen, Study on the performance of heat storage and heat release of water storage tank with PCMs, Energy and Buildings 158, 1770-1780 (2018)

9. J.P. da Cunha, P. Eames, Compact latent heat storage decarbonisation potential for domestic hot water and space heating applications in the UK, Appl. Thermal Eng. 134, 396-406 (2018)

10. Grant Agreement No. 768921 - HEART - H2020EEB-2017, (2017), https://cordis.europa.eu/project/rcn/211645 en.html

11. H. Mehling, L. F. Cabeza, Heat and Cold Storage with PCM : An up to date introduction into basucs and applications, Springer, (2008)

12. VDI 2164, PCM energy storage systems in building services, Verein Deutscher Ingenieure e.V., (2016)

13. Rubitherm, RT28HC Data sheet, https://www.rubitherm.eu/media/products/datasheets /Techdata_RT28HC EN 31052016.PDF 